Apidologie, 1977, 8 (4), 401-411.

\title{
WELCHE GRÖßEN BEEINFLUSSEN DIE MENGE DER VON BIENEN UND WESPEN AN DER FUTTERQUELLE AUFGENOMMENEN ZUCKERLÖSUNG
}

\author{
Facteurs influençant la quantité de solution de sucre récoltée \\ par les abeilles et les guêpes à la source de nourriture
}

Walter PFLUMM

Fachbereich Biologie, Postfach 3049, D-6750 Kaiserslautern

\begin{abstract}
SUMMARY
FAGTORS DETERMINING THE VOLUME OF SUGAR SOLUTION BEES

AND WASPS COLLECT PER TRIP AT THE FOOD SOURCE
\end{abstract}

With honeybees (Apis mellifica) and wasps (Paravespula germanica and P. vulgaris), the volume of load per trip as a function of different variables is described.

With bees, the volume of load per trip depends on the form of the food container (Fig. 1, inserted figure on the left).

With bees, the volume of load per trip grows with increasing concentration of the sucrose solution. With wasps, the volume of load per trip does not depend on the sucrose concentration (Fig. 2).

With bees and wasps, the volume of load per trip rises with increasing influx velocity of the solution (Fig. 3).

With bees, the volume of load per trip remains nearly constant even when the temperature of the solution varies considerably (Fig. 4, inserted figure).

With bees and wasps, the volume of load per trip grows with increasing air temperature at the food source. The slopes of the curves depend on the temperature range (Fig. 4).

With bees, the volume of load per trip depends on the size of the rectal bladder (Fig. 5).

At an artificial food source one can offer huge quantities of sugar per unit of time to a forager. With flowers, circumstances like these occur only in exceptional cases. For this reason, with bees foraging on flowers in the open, the values of the volume of load per trip are not so high as with foragers on artificial food sources (Fig. 6). 


\section{ZUSAMMENFASSUNG}

Die Abhängigkeit der Abflugmagenfüllung bei Bienen (Apis mellifica) und Wespen (Paravespula germanica und $P$. vulgaris) von verschiedenen experimentellen Variablen ist dargestellt.

Die Abflugmagenfüllung hängt bei Bienen von der Form des Safthalters ab (Abb. 1, Einschaltfigur links).

Bei Bienen wächst die Abflugmagenfüllung mit zunehmender Konzentration der Saccharoselösung. Bei Wespen hängt die Abflugmagenfüllung nicht von der Konzentration der Lösung ab (Abb. 2).

Bei Bienen! und Wespen wächst die Abflugmagenfüllung mit zunehmender Zuflußgeschwindigkeit der Lösung (Abb. 3).

Die Temperatur der Zuckerlösung hat bei Bienen nur einen geringen Einfluß auf die Abflugmagenfüllung (Abb. 4, Einschaltfigur).

Die Abflugmagenfüllung wächst bei Bienen und Wespen mit zunehmender Lufttemperatur an der Futterquelle. Die Zunahme der Abflugmagenfüllung pro Temperatureinheit ist in verschiedenen Temperaturbereichen unterschiedlich (Abb. 4). (Abb. 5).

Bei Bienen hängt die Abflugmagenfüllung vom Füllungszustand der Rektalblase ab

In einer künstlichen Futterquelle kann man einer Sammlerin sehr große Zuckermengen pro Zeiteinheit anbieten. Solche Verhältnisse kommen bei Blüten nur in Ausnahmefällen vor. Daher erreicht die Abflugmagenfüllung bei im Freien an Blüten sammelnden Bienen keine so hohen Werte wie bei Sammlerinnen an künstlichen Futterquellen (Abb. 6).

Aвв. 1. - OBEN : Künstliche Blume, kurz als «Futterquelle» bezeichnet. - EINSCHALTFIGUR RECHTS : Sammelzyklus. In der Mitte an der Futterquelle saugende Biene, schematisch. Honigmagen punktiert. Vor dem Honigmagen liegende Abschnitte des Verdauungstrakts fortgelassen. Rektalblase schraffiert. Große FigUR : Abflugmagenf üllung im Verlauf eines Tages bei gleichbleibender Reizsituation an der Futterquelle (3.8.1976). - EINSCHALTFIGUR LiNKs : Einfluß der Form des Safthalters auf die Abflugmagenfüllung. Nach KaPPEx (1953). Bei diesen Versuchen wurde die getrunkene Menge durch einen Wägevorgang festgestellt (für die Einschaltfigur wurden die Werte in $\iota_{1}$ umgerechnet).

FIg. 1. - HAUT. Fleur artificielle, désignée par le terme de "source de nourriture ".

Figure insérée a Droite. Cycle de récolte. Au milieu près de la source de nourriture, représentation shématique d'une abeille en train d'aspirer. Jabot en pointillés. Portions du tube digestif avant le jabot, non représentées. Ampoule rectale hachurée.

GRANDE FIGURE : volume de la charge de nourriture collectée à chaque voyage durant une journée, la source de nourriture restant également stimulante (3.8.1976).

FIGURE INSÉRÉE A GAUCHE : influence de la forme du récipient sur le volume de la charge collectée. D’äprès KAPpel (1953). Dans ces expériences la quantité bue était déterminée par pesée (sur la figure insérée les valeurs ont été converties en $\mu l)$. 
MENGE DER AUFGENOMMENEN ZUCKERLÖSUNG
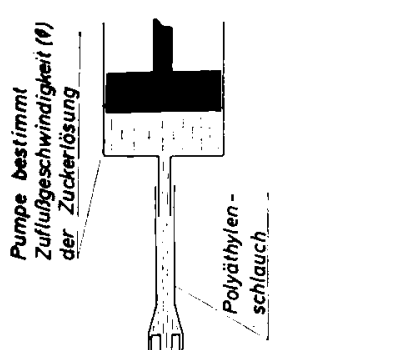$$
\text { (1) }
$$

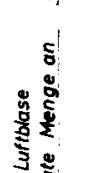
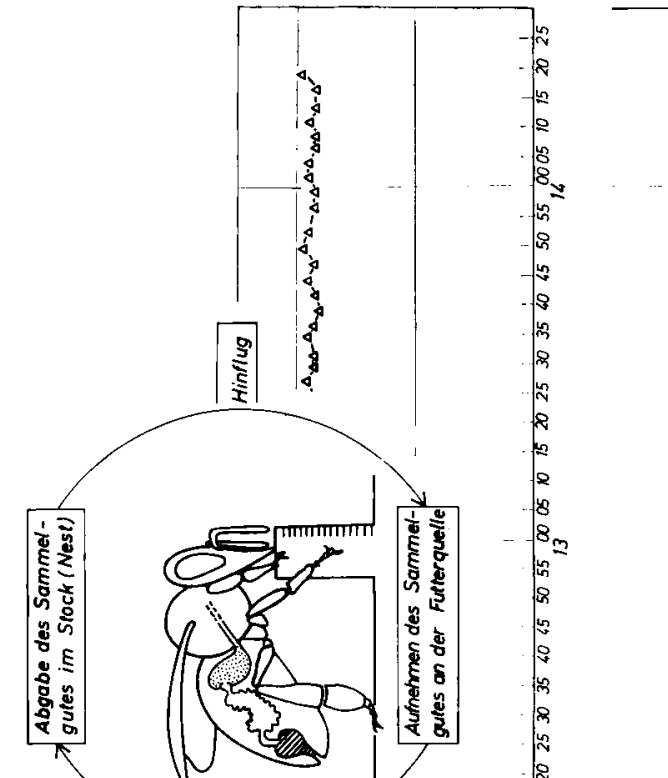
In unseren Breiten sind vorwiegend Insekten Blütenbesucher und -bestäuber (Zusammenfassung : KugLer, 1970). Sie finden in den Blüten neben Pollen Nektar. Die Besucher nehmen diese Zuckerlösung entweder nur für den individuellen Bedarf auf (z.B. Schwebfliegen) oder sie sammeln für ihr Volk (Bienen, Wespen). Will man untersuchen, wie beispielsweise die Konzentration der Zuckerlösung das Verhalten der Sammlerinnen beeinflußt, benutzt man eine künstliche Futterquelle, an der man verschiedene Größen variieren kann (Abb. 1 oben). Von diesen experimentellen Variablen hängt auch die - im folgenden behandelte - Zuckerwassermenge ab, welche eine Sammlerin bei einem Besuch an der Futterquelle bis zum Zeitpunkt des Abflugs aufnimmt (Abflugmagenfüllung). Für diesen Beitrag wurden sammelnde Insekten ausgewählt : die stark an den Blütenbesuch angepaßte Honigbiene (Apis mellifica) sowie die sozialen Faltenwespen Paravespula germanica und $P$. vulgaris.

\section{A. - TAGESABLAUF EINER NEKTARSAMMLERIN}

Das Verhalten einer Sammelbiene bzw. -wespe läuft zYкLIsch ab (Abb. 1, Einschaltfigur rechts). Flüssiges Sammelgut wird bei Bienen in der Honigblase, bei Wespen im Kropf transportiert. Beide Behälter erhalten im folgenden die Bezeichnung " Honigmagen ». Für den eigenen Stoffwechsel kann die Sammlerin Zuckerlösung aus dem Honigmagen in den Darm übertreten lassen - die Abgabe des Sammelgutes geschieht durch Auswürgen. Eine Nektarsammlerin trägt ausschließlich Nektar oder sonstige Zuckerlösungen ein. Vor dem Start zum Heimflug muß sie die Entscheidung treffen, ob sie genug gesammelt hat und aufbrechen - oder weitersaugen soll. Eine naheliegende Annahme wäre : Sie fliegt dann ab, wenn der Honigmagen voll ist. Ist dies immer der Fall?

\section{B. - MESSEN DER ABFLUGMAgenfüLLUNG; ABKÜRZUNGEN (VGL. ABB. 1 OBEN)}

Die Sammlerin landet auf dem Futtertischchen und beginnt zu saugen (= Besuchsbeginn). An der Luftblase in der Meßpipette läßt sich die bis zum Start zum Heimflug (= Besuchsende) gesaugte Menge (= Abflugmagenfüllung in $\mu l)$ ablesen. (Der von NuNez eingeführte Begriff “ Abflugmagenfüllung » wurde von RAU in " Endmagenfüllung " umgewandelt, um ihn auch auf laufende Bienen anwenden zu können. Hier wird der Ausdruck “ Abflugmagenfüllung » durchgehend gebraucht.).

Abkürzungen (nach Pflumm, 1969) :

$c$ : Konzentration der Zuckerlösung in \% (g pro $100 \mathrm{~g}$ Lösung). Außer bei dem unter D 2 angeführten Experiment mit Glucose wurde bei sämtlichen Versuchen Saccharose verwendet.

$v$ : Zuflußgeschwindigkeit der Lösung in $\mu \mathrm{l} / \mathrm{sec}$.

$v_{r}$ : Relative Zuflußgeschwindigkeit. Dimensionslos, da bezogen auf die maximale Sauggeschwindigkeit $\left(v_{\max }\right)$ der Sammlerin bei der betreffende 1 Temperatur. Kann sie trinken so schnell sie vermag, wird dies als “ voller Zufluß » bezeichnet.

$T$ : Luftemperatur an der Futterquelle in ${ }^{\circ} \mathrm{C}$. 


\section{C. - AbFlugmagenfüllung als meßgrößE}

Wenn sich die Reizsituation an der Futterquelle nicht ändert, mißt man bei jedem Besuch die gleiche Abflugmagenfüllung. Abb. 1 zeigt neben dieser Konstanz der Abflugmagenfüllung die große Regelmäßigkeit, mit der eine Sammlerin eine Futterquelle besucht.

\section{D. - ABFLUGMAGENFÜLLUNG IN ABHÄNGIGKEIT VON VERSCHIEDENEN GRÖßEN}

Bei den im folgenden beschriebenen Versuchen wurde jeweils eine experimentelle Variable verändert, während die übrigen konstant gehalten wurden.

\section{1. - Form des « Safthalters »}

Beim Saugen aus Uhrglas-Schälchen ist bei Bienen die Abflugmagenfüllung geringer als beim Trinken aus Kapillaren (Abb. 1, Einschalt figur links). Ob für Wespen Entsprechendes gilt, ist eine offene Frage. Bei sämtlichen im folgenden geschilderten Experimenten wurden die Zuckerlösungen den Sammlerinnen in Kapillaren geboten.

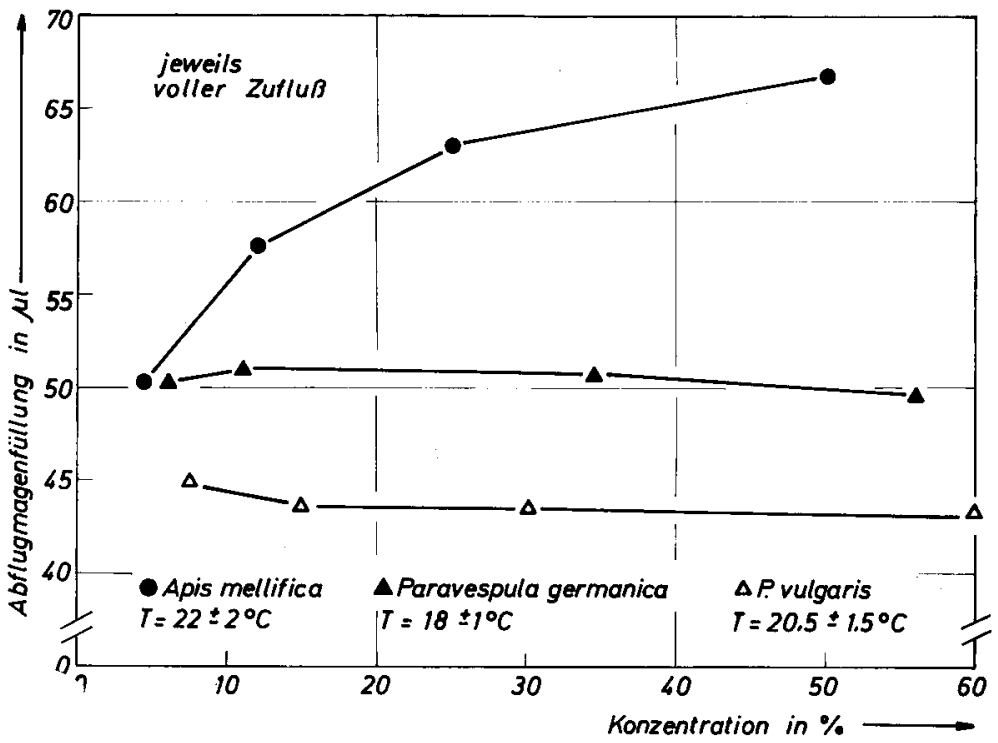

Aвв. 2. - Abflugmagenfüllung als Funktion der Konzentration. Werte für die Biene und P. germanica übernommen aus PfLum $(1975 ; \mathrm{Abb} .2)$; dabei wurden für die Wespen für entsprechende Ảbszissenwerte jeweils gemeinsame Mittelwerte errechnet. $P$. vulgaris : Unveröffentlichte Meßwerte. September 1976. 1 Wespe. Mittelwerte. $<15 \mathrm{n}<47$.

Fıc. 2. - Volume de la charge collectée en fonction de la concentration. Les valeurs pour l'abeille et $P$. germa. nica proviennent de Pflumm (1975, fig. 2); pour les guêpes on a calculé les valeurs moyennes générales pour les valeurs d'abscisses correspondantes. $P$. vulgaris : mesures non publiées. Septembre 1976.1 guêpe. Moyennes $15<\mathrm{n}<47$. 


\section{2. - Konzentration}

Die Sammlerin stellt die Konzentration mit Hilfe des Geschmackssinnes fest. Wie Abb. 2 zeigt, füllen die Wespen ihren Honigmagen immer gleich stark - unabhängig von der Konzentration (Lit. s. Legende Abb. 2). Die Bienen dagegen kehren bei niedriger Konzentration mit nur zu drei Vierteln gefülltem Honigmagen zum Stock zurück; bei ihnen wächst die Abflugmagenfüllung mit zunehmender Konzentration (BetTs, 1929; v. Frisch, 1935, 1965; Gontarski, 1935; Nunez, 1966; Pflumm, 1969).

Dies gilt nicht nur für Saccharose-, sondern auch für Glucoselösungen (v. Frisch, 1935). Zur Diskussion der diesen Angaben widersprechenden Befunde von Wells und Giacchino (1968) vgl. Pflum (1969, S. 7-8).

Bei sehr hoch konzentrierter Nahrung (z.B. festem Zucker), welche die Bienen LeCKend-saugend (unter Zugabe von Pharynxdrüsensekret) aufnehmen müssen, ist die Abflugmagenfüllung geringer als bei flüssiger, welche aufgeSaUgT werden kann (Gontarski und Hoffmann, 1963).

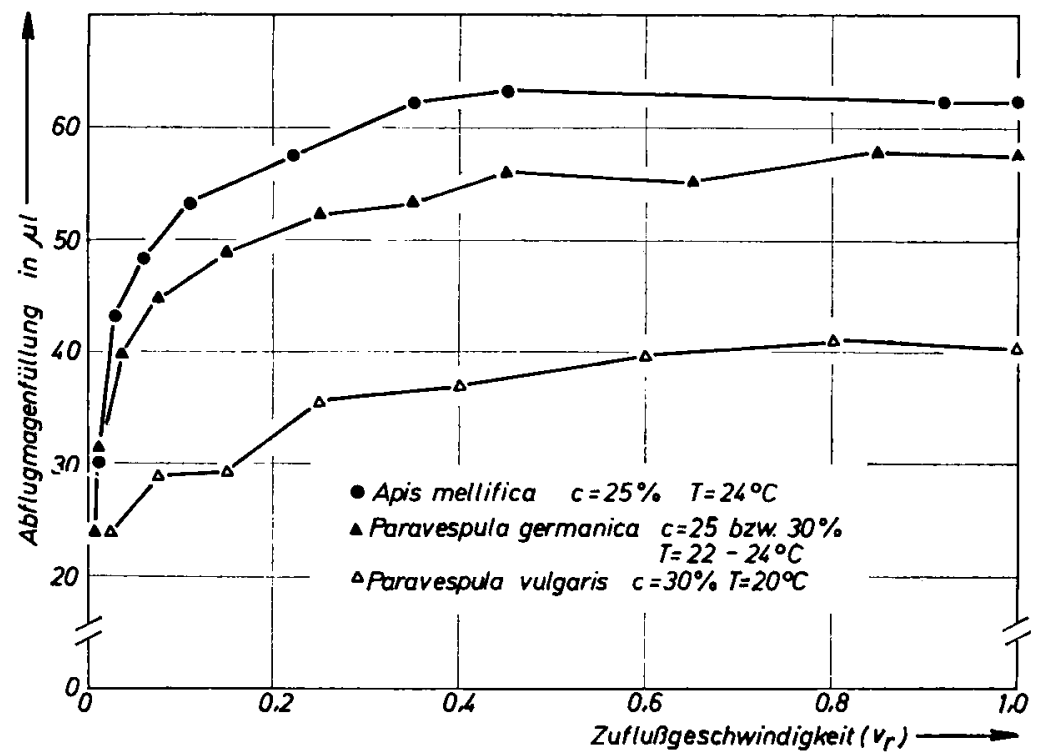

Aвв. 3. - Abflugmagenfûllung in Abhängigkeit von der Zuflußgeschwindigkeit. Werte aus Abb. 1 bei Prlumm (1971) übernommen. Aus den Werten der 3 Individuen von $P$. germanica wurden jeweils gemeinsame Mittelwerte errechnet. $v_{\max }$ betrug unter den angegebenen Bedingungen : 1,52 $\mu \mathrm{l} / \mathrm{sec}$ für die Biene (Mittelwert von 7 Individuen); 1,71 $\mu \mathrm{l} / \mathrm{sec}$ für $P$. germanica (Mittelwert von 3 Individuen); $1,0 \mu 1 / \mathrm{sec}$ für $P$. vulgaris (1 Individuum).

FIg. 3. - Volume de la charge collectée en fonction de la vitesse de l'aflux. Les valeurs sont tirées de la figure 1 de Prlumm (1971). A partir des valeurs des 3 individus de $P$. germanica on a calculé les valeurs moyennes générales. Dans les conditions indiquées $v_{\max }$ s'élève à $1,52 \mu \mathrm{l} / \mathrm{sec}$ pour l'abeille (moyenne de 7 individus), à à $1,71 \mu \mathrm{l} / \mathrm{sec}$ pour $P$. germanica (moyenne de 3 individus) et à $1,0 \mu \mathrm{l} / \mathrm{sec}$ pour $P$. vulgaris (1 individu). 


\section{3. - Zuflußgeschwindigkeit}

Dies war die erste Größe, deren Einfluß auf die Abflugmagenfüllung beschrieben wurde (v. Frisch, 1923). NunEz (1966) hat sie dann ausführlicher untersucht. Verringert man die Zuflußgeschwindigkeit, kann die Sammlerin nicht mehr saugen, so schnell sie vermag. In Abb. 3 ist die Zuflußgeschwindigkeit nicht in $\mu \mathrm{l} / \mathrm{sec}$, sondern in relativen Einheiten angegeben. Grund : Schiebt man mit der Pumpe das Zuckerwasser gerade so schnell nach, wie es die Sammlerin aufnehmen kann, mißt man eine bestimmte Zuflußgeschwindigkeit, die mit der Sauggeschwindigkeit der Sammlerin identisch ist $\left(v_{\max }\right)$. Unter diesen Bedingungen saugen verschiedene Sammlerinnen verschieden schnell. Um einhcitliche Kurven zu erhalten, wurde für jede Sammlerin $v_{\max }=1$ gesetzt und jede kleinere Zuflußgeschwindigkeit auf diesen Wert bezogen ( $v_{r}$-Skala). Wie Abb. 3 zeigt, bleibt die Abflugmagenfüllung mit abnehmender Zuflußgeschwindigkeit zunächst etwa konstant; unterhalb $0,4<\mathrm{v}_{\mathrm{r}}<0,5$ beginnen die Kurven zu fallen, ab etwa $\mathrm{v}_{\mathrm{r}}=0,1$ streben sie rasch gegen Null. Dies gilt für Bienen und Wespen.

\section{4. - Temperatur des Zuckerwassers}

Die Abflugmagenfüllung wird bei Bienen von der Temperatur der Lösung kaum beeinflußt (AJ b. 4, Einschaltfigur).

\section{5. - Luftem;eratur an der Futterquelle}

Im Gegensatz zur Tempera rur der Lösung übt die Lufttemperatur einen Einfluß auf die Abflugmagenfüllung aus (Bienen : Betrs, 1929; v. Frisch, 1935; Gontarski, 1935; Schuà, 1952; Nùñez, 1966). Wie Abb. 4 zeigt, wächst bei Bienen die Abflugmagenfüllung stark mit zunehmender Temperatur, aber nur bis zu einem Wert von etwa $21^{\circ} \mathrm{C}$; oberhalb dieses Wertes ist die Zunahme der Abflugmagenfüllung pro Temperatureinheit geringer als darunter. Bei Wespen ist eine starke Abhängigkeit der Abflugmagenfüllung von der Temperatur nur bei sehr tiefer Temperatur zu beobachten, von $6^{\circ} \mathrm{C}$ bis etwa $19^{\circ} \mathrm{C}$ steigt die Kurve langsam, oberhalb $19^{\circ} \mathrm{C}$ bleibt die Abflugmagenfüllung konstant.

\section{6. - Füllungszustand der Rektalblase}

Diese Abhängigkeit wurde bei Bienen untersucht. Wenn eine Biene am Besuchsende von der Futterquelle abfliegt, beobachtet man häufig, daß sie kurz nach dem Start Rektalblasen-Flüssigkeit abgibt (Lit. bei Pasedach- 
Poeverleiv, 1941). Zwingt man die Bienen, den Weg zwischen Stock und Futterquelle laufend zurückzulegen, vermögen sie offenbar die Rektalblase nicht zu entleeren. Mit zunehmender Rektalblasenfüllung sinkt die Abflugmagenfüllung. Wird die Rektalblase in einem " Reinigungsflug 》 entleert, steigt die Abflugmagenfüllung sprunghaft an (RAU, 1970). Ein solches langsames Abfallen und sprunghaftes Ansteigen der Abflugmagenfüllung (vgl. RAU,

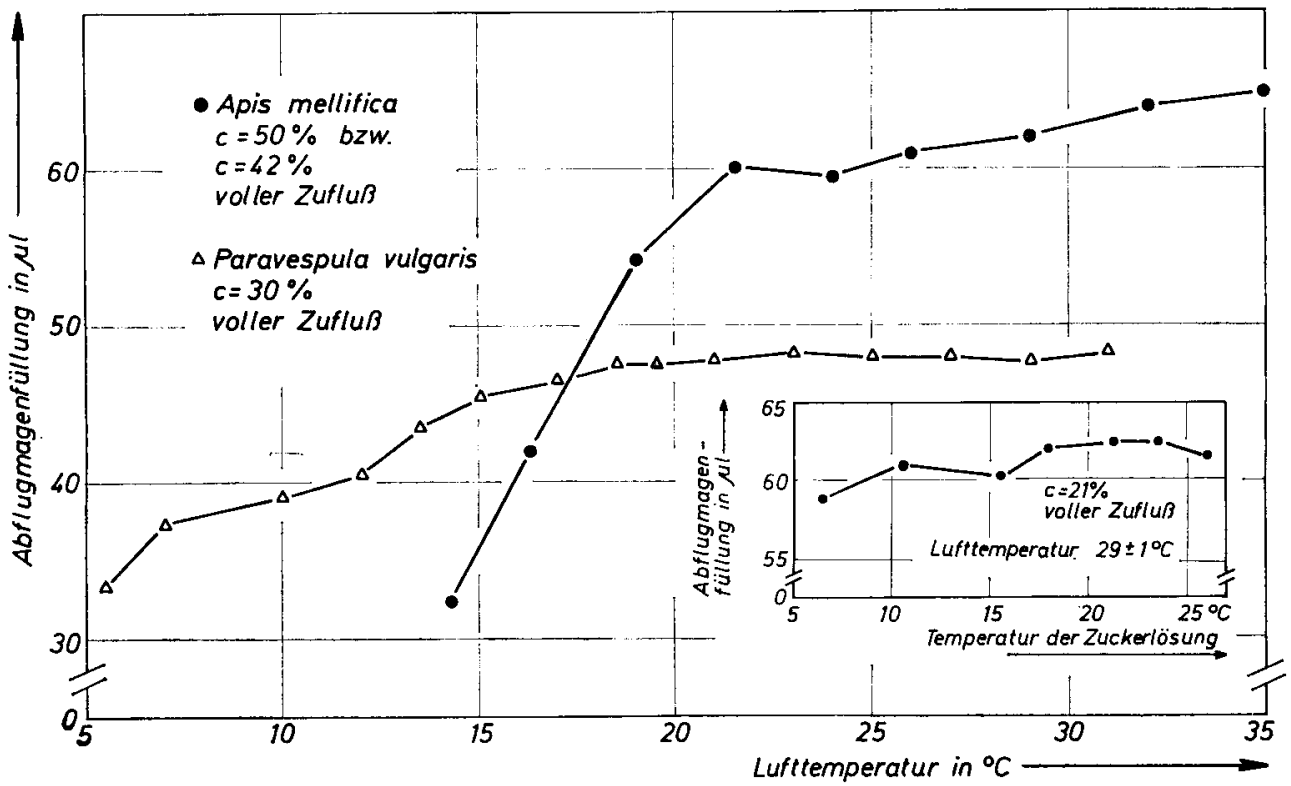

Aвв. 4. - Abflugmagenfüllung als Funktion der Luftemperatur an der Futterquelle. Werte für Apis übernommen aus NUNEz (1966; Abb. 9. Bienen « WEISS 》 und « RoT », $\mathrm{c}=42 \%$ ); ergänzt durch eigene Meßwerte vom September 1965 (unveröffentlicht; 1 Biene; $c=50 \%$; zum Einstellen der Temperatur vgl. Pruumm, 1969). Für jede Biene wurden aus den je innerhalb eines Temperaturbereichs von $2{ }^{\circ} \mathrm{C}$ liegenden Meßwerten Mittelwerte errechnet $(2<\mathrm{n}<28)$. Diese wurden auf den jeweiligen Mittelwert bei $32{ }^{\circ} \mathrm{C}$ normiert (gemeinsamer Mittelwert der 3 Bienen bei $32^{\circ} \mathrm{C}: 64,1 \mu \mathrm{l}$ ). Bei einer in der Abbildung nicht dargestellten Biene (Juni 1966; $c=50 \%$ ) prüfte ich (PFLumm, 1968) den Unterschied zwisehen den Werten bei $20^{\circ} \mathrm{C}$ und $30^{\circ} \mathrm{C}$ mit dem $t$-Test; die Differenz ist signifikant $: P<0,001$. - Werte für $P$. vulgaris nach Prudm (in Vorbereitung a). August/September 1976. 4. Wespen. Bearbeitung der Daten wie für die Bienen geschildert, nur Mittelwerte hier auf den jeweiligen Mittelwert bei $18{ }^{\circ} \mathrm{C}$ normiert (gemeinsamer Mittelwert der 4 Wespen bei $18{ }^{\circ} \mathrm{C}: 47,6$ ul). - ErNschaltFigur : Abflugmagenfüllung in Abhängigkeit von der Temperatur der Zuckerlösung. Zusammengefaßte Meßwerte aus Abb. 7 bei NunEz (1966).

Fig. 4. - Volume de la charge collectêe en fonction de la température de l'air à la source de nourriture. Les valeurs pour $A$ pis sont tirées de Nunez (1966; fig. 9, abeilles « blanche » et « rouge », c $=42 \%$ ) et complétées par des mesures personnelles de septembre 1965 (non publiées; 1 abeille; c $=50 \%$; pour le réglage des températures, voir Pfuumm, 1969). Pour chaque abeille on a calculé les moyennes à partir de mesures faites dans des domaines de température variant de $2{ }^{\circ} \mathrm{C}(2<\mathrm{n}<28)$. Elles ont été rapportées à chaque moyenne à $32{ }^{\circ} \mathrm{C}$ (moyenne générale des 3 abeilles à $32{ }^{\circ} \mathrm{C}: 64, \mathrm{I} \mu \mathrm{l}$ ). Chez l'une des abeilles non représentées sur la figure (juin 1966, $\mathrm{c}=50 \%$ ), j'ai contrôlé la différence entre les valeurs à $20^{\circ} \mathrm{C}$ et $30^{\circ} \mathrm{C}$ par le test-t; la différence est significative $: \mathbf{P}<0,001$. Les valeurs pour $P$. vulgaris proviennent de PfLUMM (en préparation, a). Août/septembre 1976. 4 guêpes. Le traitement des données est le même que celui décrit pour les abeilles, mais les moyennes ont été rapportées ici à chaque moyenne à $18^{\circ} \mathrm{C}$ (moyenne générale des 4 guêpes à $\left.18{ }^{\circ} \mathrm{C}: 47,61\right)$.

Figure InsÉrée : volume de la charge collectée en fonction de la température de la solution de sucre. Mesures tirées de la figure 7 de NunEz (1966). 
1970; Abb. 9) wurde auch bei fliegenden Bienen in einem Flugraum beobachtet — nie jedoch bei Freilandversuchen (Abb. 5). Möglicherweise spielt dabci die Entfernung zwischen Futterquelle und Stock eine Rolle.

\section{E. - BLÜTENÖKOLOGISCHER ASPEKT (DIE AUSSAgEN DIESES ABSCHNITTS GELTEN FÜR BIENEN)}

Will man die an der künstlichen Futterquelle erhobenen Befunde auf Blüten übertragen, bringt man Konzentration und Zuflußgeschwindigkeit auf einen “ gemeinsamen Nenner » : Durch Multiplikation der beiden Größen erhält man die von der Sammlerin in der Zeiteinheit gewonnene Zuckermenge. Die Biene dürfte die beiden Größen auf dieselbe Weise verrechnen (PfLumm, 1970 a und in Vorbereitung b).

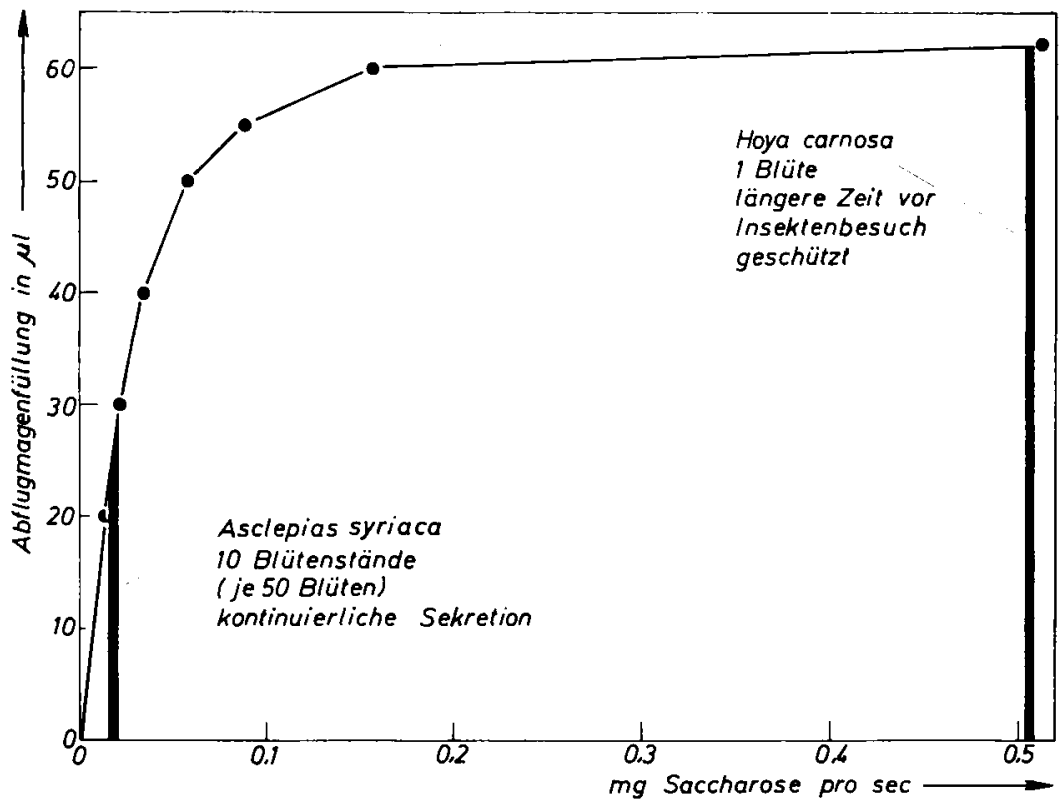

Aв8. 6. - Abflugmagenfüllung als Funktion der von der Futterquelle pro Zeiteinheit gespendeten Zuckermenge. Werte aus Abb. $17\left(\mathrm{c}=59 \% ; \mathrm{T}=24{ }^{\circ} \mathrm{C}\right)$ bei NuNEZ (1966) übernommen. Daten für Asclepias aus BEUTLER (1930) und Kleber (1935), für Hoya aus Pflum (1970 a). Zucker des Asclepias-Nektars (c $=58 \%$ ) nahezu ausschließlich Saccharose (BEUTLER, 1930). Trockensubstanz des Hoya-Nektars (c $=30 \%$; im betreffenden Experiment eingedickt : $c=60-70 \%$ ) zu fast $90 \%$ aus Saccharose bestehend (Lit. bei Pflumm, 1970 a).

Fic. 6. - Volume de la charge collectée en fonction de la quantité de sucre distribuée à la source de nourriture par unités de temps. Valeurs tirées de la figure $17\left(\mathrm{c}=59 \% ; \mathbf{T}=24^{\circ} \mathrm{C}\right)$ de NunEz $(1966)$. Les données concernant Asclepias proviennent de Beutler (1930) et de KLeber (1935), celles concernant Hoya de Pflumm $(1970 \mathrm{c})$. Le sucre du nectar d'Asclepias $(\mathrm{c}=58 \%$ ) est presqu'uniquement du saccharose (Beutler 1930). La matière sèche du nectar de Hoya $(c=30 \%$; dans l'expérience en question, augmentée à $c=60$ $70 \%$ ) est composée à près de $90 \%$ de saccharose (bibliogr. de Pfuumm, 1970 a). 
Wie Abb. 6 zeigt, spendet selbst die ergiebigste von Beutler (1930) und Kleber (1935) untersuchte Nektarpflanze Asclepias syriaca $(=A$. cornuti) sehr wenig Zucker pro Zeiteinheit. Große Zuckermengen - wie in der künstlichen Futterquelle - gibt es bei Blüten nur in Ausnahmefällen (Abb.6). Wird bei fortwährendem Beflug der sezernierte Nektar gleich weggesaugt, muß eine Biene während eines Sammelflugs eine große Anzahl Blüten abernten : Eine Sammlerin besuchte während eines 1 Stunde 51 Minuten dauernden Aufenthalts am Futterplatz rund 3800 Blüten von Heracleum sphondylium (Pflumm, 1970 b).

Eingegangen im Mai 1977.

Reçu pour publication en mai 1977.

\section{RESUMÉ}

On décrit la relation entre le volume de la charge de nourriture, collectée à chaque voyage par les abeilles (Apis mellifica) et les guêpes (Paravespula germanica et $P$. vulgaris), et différentes variables.

Chez les abeilles le volume de la charge collectée dépend de la forme du récipient (Fig. 1, figure insérée à droite); il augmente avec la concentration de la solution; chez les guêpes il ne dépend pas de la concentration de la solution (Fig. 2).

Chez les abeilles comme chez les guêpes le volume de la charge collectée augmente avec la vitesse de l'afflox (Fig. 3).

La température de la solution de sucre n'a qu'une faible influence sur le volume de la charge collectée par les abeilles (Fig. 4, figure insérée).

Chez les abeilles et les guêpes, le volume de la charge collectée augmente avec la température de l'air à la source de nourriture. L'augmentation varie selon le domaine de température (Fig. 4).

Le volume de la charge collectée dépend chez les abeilles de l'état de remplissage de l'am poule rectale (Fig. 5).

A une source artificielle de nourriture il est possible d'offrir à une butineuse d'énormes quantités de sucre par unités de temps. De telles conditions ne se rencontrent qu'exceptionnellement avec les fleurs. Il s'ensuit que le volume de la charge collectée n'atteint pas des valeurs aussi fortes chez les abeilles qui butinent sur des fleurs en plein air que chez celles qui butinent à une source artificielle de nourriture.

\section{LITERATUR}

Betrs A., 1929. - Das Aufnahmevermögen der Bienen beim Zuckerwasserfüttern. Arch. Bienenk., 10, 301-309.

BeUtler R., 1930. - Biologisch-chemische Untersuchungen am Nektar von Immenblumen. Z. vergl. Physiol., 12, 72-176.

Frisch K. v., 1923. - Über die “ Sprache " der Bienen, eine tierpsychologische Untersuchung. Zool. Jb., Abt. allg. Zool. u. Physiol. 40, 1-186.

Frisch K. v., 1935. - Über den Geschmackssinn der Biene. Z. vergl. Physiol, 21, 1-157.

Frisch K. v., 1965. - Tanzsprache und Orientierung der Bienen. Springer, Berlin-HeidelbergNew York, $578 \mathrm{~S}$. 
Gontarski H., 1935. - Leistungsphysiologische Untersuchungen an Sammelbienen (Apis mellifica). Arch. Bienenk., 16, 107-126.

Gontarski H. und Hoffmann J., 1963. - Über Aufnahme und Verarbeitung der zuckerhaltigen Nahrung bei der Honigbiene. Z. Bienenf., 6, 184-198.

Kappel I., 1953. - Die Form des Safthalters als Anreiz für die Sammeltätigkeit der Bienen. Z. vergl. Physiol., 34, 539-546.

Kleber E., 1935. - Hat das Zeitgedächtnis der Bienen biologische Bedeutung? Z. vergl. Physiol., 22, 221-262.

KUgler H., 1970. - Blütenökologie. Fischer-Stuttgart, 345 S.

N: NEz J. A., 1966. - Quantitative Beziehungen zwischen den Eigenschaften von Futterquellen und dem Verhalten von Sammelbienen. Z. vergl. Physiol., 53, 142-164.

Pasedach-Poeverlein K., 1941. - Über das " Spritzen " der Bienen und über die Konzentrationsänderung ihres Honigblaseninhalts. Z. vergl. Physiol., 28, 197-210.

Pflcim W., 1968. - Zum Verhalten nektarsammelnder Honigbienen an der Futterquelle. Disscrtation Math.-Naturw. Fak. Univers. Freiburg i. Br.

Pflumm W., 1969. - Beziehungen zwischen Putzverhalten und Sammelbereitschaft bei der Honigbiene. Z. vergl. Physiol., 64, 1-36.

Pflum W., 1970 a. - Auslösende Faktoren beim Duftmarkieren von Sammelbienen. Experientia (Basel), 26, 318-319.

Pflumm W., 1970 b. - Beobachtungen zum Putzverhalten nektarsammelnder Honigbienen. Zool. Jb., Abt. allg. Zool. u. Physiol., 75, 319-324.

Pflc мм W., 1971. - Zum Sammelverhalten von Wespen. Insectes sociaux, 18, 155-160.

Pflum W., 1975. - Einfluß verschiedener Zuckerwasser-Konzentrationen und der Anwesenheit von Artgenossen auf das Sammelverhalten von Wespen (Hymenoptera : Vespidac). Ent. Germ., 2, 7-21.

Pflumm W. - Flugaktivität und Sammelverhalten von Wespen (Paravespula vulgaris) in Abhängigkeit von Beleuchtungsstärke und Temperatur (in Vorbereitung a).

Pflumm W. - Die Bildung der Führungsgröße für die Abflugmagenfüllung der Sammelbiene (in Vorbereitung b).

RAU G., 1970. - Zur Steuerung der Honigmagenfüllung sammelnder Bienen an einer künstlichen Futterquelle. Z. vergl. Physiol., 66, 1-21.

SchUA L., 1952. - Untersuchungen über den Einfluß meteorologischer Elemente auf das Verhalten der Honigbienen (Apis mellifica). Z. vergl. Physiol., 34, 258-277.

Wells P. H., Giacchino J. Jr., 1968. - Relationship between the volume and the sugar concentration of loads carried by honeybees. J. Apicult. Res., 7, 77-82. 